\title{
ENCUENTROS Y DESENCUENTROS EN LA EDUCACIÓN INTERCULTURAL BILINGÜE: CASO CECIB IKA Y TRÁNSITO AMAGUAÑA
}

\section{ENCOUNTERS AND DISAGREEMENTS IN BILINGUAL INTERCULTURAL EDUCATION: CECIB IKA AND TRÁNSITO AMAGUAÑA CASE}

DINORA HIDALGO ${ }^{1}$

ALEXANDRA YÉPEZ ${ }^{2}$

Recibido: 18 de junio de 2018 Aceptado: 22 de octubre de 2018

\footnotetext{
${ }^{1}$ Pontificia Universidad Católica del Ecuador, GHIDALGO711@puce.edu.ec

${ }^{2}$ Pontificia Universidad Católica del Ecuador, SYEPEZ838@puce.edu.ec
} 
illllil15 


\section{ENCUENTROS Y DESENCUENTROS EN LA EDUCACIÓN INTERCULTURAL BILINGÜE: CASO CECIB IKA Y TRÁNSITO AMAGUAÑA}

\section{ENCOUNTERS AND DISAGREEMENTS IN BILINGUAL INTERCULTURAL EDUCATION: CECIB IKA AND TRÁNSITO AMAGUAÑA CASE}

\section{Dinora Hidalgo, Alexandra Yépez}

Palabras clave: educación intercultural, encuentros, desencuentros, contextos.

Keywords: intercultural education, encounters, disagreements, contexts.

\section{RESUMEN}

El artículo presenta una recopilación breve sobre los enunciados de principios, fines, objetivos, de las instituciones rectoras de la educación intercultural. Posteriormente, se evidencia la etnografía realizada en un Centro Educativo Comunitario Intercultural Bilingüe CECIB Ika, de la Amazonía ecuatoriana que atiende a la niñez waorani, y un acercamiento a la Unidad Educativa Tránsito
Amaguaña que atiende a niños kichwa de la sierra ecuatoriana. La investigación procura describir los principios de la interculturalidad en el ámbito de la educación e identificar los encuentros y desencuentros en la aspirada interculturalidad de la educación intercultural bilingüe. Los objetivos pretenden responder a la pregunta de investigación: ¿Cómo se llega a plasmar en la realidad de los Centros 
de Educación Comunitaria Intercultural Bilingüe (CECIB) uno de los derechos de la niñez, la educación, sobre todo en sociedades multiculturales y multiétnicas? Los resultados de la investigación lleva- ron a reflexiones plasmadas en las conclusiones, pudiéndose manifestar que hay más desencuentros que encuentros a la hora de educar en contextos interculturales bilingües.

\section{ABSTRACT}

The article presents a brief compilation regarding the statements of principles, aims and objectives of the governing institutions of intercultural education. The ethnography carried out in a Bilingual Intercultural Community Educational Center CECIB Ika -which is in the Ecuadorian Amazon and serves Waorani children and an approach to the Educational Unit Transit Amaguaña that serves Kichwa children of the Ecuadorian highlands are then shown. The purpose of the research is to describe the principles of interculturality in the field of education and to identify the encounters and disagreements in the intercultural aspirations of intercultural bilingual education. The objectives are intended to answer the following research question: how can one of the rights of children - education - in particular in multicultural and multi-ethnic societies, be translated into the reality of the Centers for Intercultural Bilingual Community Education (CECIB)? The results of the research led to reflections that are shown in the conclusions. It can be stated that there are more disagreements than points of agreement when it comes to educating in bilingual intercultural contexts.

\section{METODOLOGÍA}

Para aproximarnos a una realidad inquietante a nuestro quehacer profesional como antropólogas, psicólogas y pedagogas, recurrimos al método etnográfico, a través de la investigación de tipo exploratorio, estudio de caso, realizada en dos escuelas consideradas dentro del sistema de gestión de la educación intercultural bilingüe EIB, a estos centros hoy se los conoce como Centros Comunitarios Interculturales Bilingüe CECIB, y también, escuelas guardianas de la lengua.

La investigación toma de referencia al CECIB IKA, ubicado en el Par- 
que nacional Yasuní, espacio geográfico compartido entre varios actores, como es el caso de la Estación científica de la Pontificia Universidad Católica del Ecuador, la petrolera Repsol y una parte de la población waorani; y a la Unidad Educativa Tránsito Amaguaña, ubicada en la ciudad de Quito, centro educativo que trabaja con población indígena de la nacionalidad kichwa y atiende en su mayoría a los niños de las familias que trabajan en el mercado mayorista.

La etnografía consistió en observaciones a la realidad, tanto de los espacios como de los comportamientos y actividades de las personas adultas y niños que construyen los contextos educativos, conversaciones mediante entrevistas con directores, maestros y miembros de la comunidad, trabajos en grupos focales, es decir, se dio un acercamiento al hecho educativo que se da en estos lugares.

Posteriormente, con la realidad captada a través de varios instrumentos, se procedió a analizar lo que se pretende con la educación intercultural bilingüe desde postulados de la Unesco, la Constitución de la República del Ecuador, la Ley Orgánica de Educación Intercultural Bilingüe del Ecuador, y lo que se evidencia en los centros que intentan aplicarla.

\section{Participantes:}

En el CECIB Ika se trabajó con 4 docentes, 50 estudiantes, con padres de familia, y miembros de la comunidad entre quienes participaron los jefes y dirigentes de las comunidades. Por otro lado, se mantuvieron diálogos con actores externos como miembros de la Estación Científica Yasuní de la PUCE, relacionadores comunitarios de Repsol, y voluntarios.

La Unidad Educativa Tránsito Amaguaña atiende a 100 familias indígenas de bajos recursos en riesgo de vulnerabilidad y funciona al interior del mercado mayorista de Quito. Ahí se realizaron entrevistas a 4 profesores, 2 directivos y 6 estudiantes de la Unidad Educativa. No se trabajó con padres de familia ni con actores externos.

\section{DESARROLLO}

\section{Antecedentes:}

El presente artículo nace de la investigación "Escuela Waorani: Entrelazando Educación y Cultura" realizada du- rante 15 meses en los años 2016 y 2017 y complementado con la investigación a realizarse en los años 2018 y 2019 "EI rol del docente en el desarrollo socioe- 
ducativo y sociocultural del Centro Comunitario Intercultural Bilingüe IKA de la población waorani" investigaciones que contaron con el aval y financiamiento de la Pontificia Universidad Católica del Ecuador y el apoyo de la Estación Científica Yasuní de la PUCE, para trabajar en el Centro Comunitario de Educación Intercultural Bilingüe (CECIB) IKA ubicado en la parroquia Labaka en la amazonía ecuatoriana, que trabaja con niños mayoritariamente de nacionalidad waorani de las comunidades de Guiyero, Timpoka, Ganketapare y Peneno.

\section{Multiculturalidad e Interculturalidad}

Parafraseando a Verónica Hidalgo (2017), y tomando en cuenta la etimología de cada término, podemos hacer una primera distinción: multiculturalidad refiere a la presencia de varias culturas en un espacio determinado sin mayor relación entre las mismas, mientras que hablar de interculturalidad nos plantea una relación de intercambio entre las diversas culturas y, por lo tanto, de su enriquecimiento mutuo.

En torno a este término se han creado una serie de miradas y de propuestas. Esto nos lleva a pensar en los principios de la Interculturalidad en las sociedades multiculturales y en las reflexiones vivenciales de la realidad, en el caso de las escuelas interculturales y bilingües. Las reflexiones que pretendemos abordar se enmarcan en lo que se puede llamar "los encuentros y los desencuentros" en esta realidad.

En el Ecuador -gracias a la iniciativa de grupos indígenas- se logró el reconocimiento de la interculturalidad. Tuvo su origen entre los años treinta y sesenta como un reclamo por la falta de condiciones de justicia. Luego, se transformó en demanda de derechos. En 1998, la Constitución declara al Estado como pluricultural y multiétnico, y, con ello, se reconoce la necesidad de atender a los pueblos y nacionalidades en ámbitos de intervención primaria: salud y educación (Krainer, 2016). Aunque, hasta el momento, aún no se evidencie igualdad de condiciones y oportunidades para los pueblos indígenas ni en salud ni en educación.

El Ministerio de educación pretende fortalecer la Educación Intercultural Bilingüe con la Ley Orgánica de Educación Intercultural (LOEI), como una forma de dar respuesta a la diversidad étnica y cultural, ahora reconocidas en el país. De esta manera, la educación intercultural, como elemento de ejecución de la educación inicial, básico y medio son el fundamento de la LOEl en el Ecuador.

Con esta idea general, se torna necesario efectuar una breve recopilación de aquello que se establece en las 
instituciones que direccionan la educación indígena, sin dejar de mencionar aquello que sirve de apoyo a las organizaciones y movimientos indígenas, esto es, el Convenio 169 de la Organización Internacional de Trabajo (OIT). El Convenio fue firmado en 1989 por un gran número de Estados latinoamericanos y tiene relevancia en la defensa de los derechos indígenas. Lo significativo y direccionador de este Convenio consiste en que los programas de educación "deberán abarcar su historia, sus conocimientos y técnicas, sus sistemas de valores" $y$, además, "deberán adoptarse disposiciones para preservar las lenguas indígenas".

En lo referente a la interculturalidad, se menciona que es un concepto dinámico y se presenta a las relaciones evolutivas entre grupos culturales. Así, el Art. 8 de la Convención sobre protección y promoción de la diversidad de las expresiones culturales Unesco 2005 señala: "la presencia e interacción equitativa de diversas culturas y la posibilidad de generar expresiones culturales compartidas, adquiridas por medio del diálogo y de una actitud de respeto mutuo".

Estos momentos de interacción con la pretendida interculturalidad en muchas ocasiones causan conflicto. Amani (2004) lo plantea así en su libro La escuela intercultural: regulación de conflictos en contextos multiculturales, por lo que se propone que estos espa- cios deben construirse desde los actores involucrados y no desde las instituciones en las que se promulgan políticas, leyes y reglamentos.

\section{Principios de la interculturalidad en la educación}

La UNESCO, en el documento denominado Directrices de la UNESCO sobre la educación intercultural, en la sección Educación, menciona las directrices sobre educación intercultural y manifiesta una serie de principios que pretenden orientar la acción en el manejo de la interculturalidad, estos principios son tres:

Principio I: la educación intercultural respeta la identidad cultural del educando impartiendo a todos una educación de calidad que se adecúe y adapte a su cultura.

Principio II: la educación intercultural enseña a cada educando los conocimientos, las actitudes y las competencias culturales necesarias para que pueda participar plena y activamente en la sociedad.

Principio III: la educación intercultural enseña a todos los educandos los conocimientos, actitudes y las competencias culturales que les permiten contribuir al respeto, el entendimiento y la solidaridad entre individuos, 
entre grupos étnicos, sociales, culturales y religiosos y entre naciones.

(p. 34)

Cada uno de estos principios cuenta con lineamientos y sugerencias para que puedan ser aplicables al momento de ejecutarse la educación.

En la Constitución del la República del Ecuador 2008, en el Art. 1, se menciona"El Ecuador es un Estado constitucional de derechos y justicia social, democrático, soberano, independiente, unitario, intercultural, plurinacional y laico. Se organiza en forma de república y se gobierna de manera descentralizada"; mientras que en el Título II Derechos, Capítulo segundo, Sección quinta: Educación, Art. 28: (...) "Es derecho de toda persona y comunidad interactuar entre culturas y participar en una sociedad que aprende. El Estado promoverá el diálogo intercultural en sus múltiples dimensiones".

Con el mandato de la constitución, el Ministerio de Educación, promulga la Ley Orgánica de Educación Intercultural y enuncia los Principios Generales, en su Art. 2.- Principios, literal z) Interculturalidad y plurinacionalidad.La interculturalidad y plurinacionalidad garantizan a los actores del Sistema el conocimiento, el reconocimiento, el respeto, la valoración, la recreación de las diferentes nacionalidades, culturas y pueblos que conforman el Ecuador y el mundo; así como sus saberes ancestrales, propugnando la unidad en la diversidad, propiciando el diálogo intercultural e intracultural, y propendiendo a la valoración de las formas y usos de las diferentes culturas que sean consonantes con los derechos humanos.

Una vez que se cuenta con un marco normativo donde se reconoce la plurinacionalidad y se asume un Estado intercultural, el Ministerio de Educación en miras de atender a esa realidad, reconociendo nacionalidades y pueblos que habitan en el territorio, poseedores de lengua, saberes, territorios, etc., que son minoría dentro de la población ecuatoriana, pero que a la vez forman parte de esa riqueza de diversidad cultural, crea el Sistema de Educación Intercultural Bilingüe (SEIB), parte sustancial del Sistema Nacional de Educación a través de la Subsecretaría de Educación Intercultural Bilingüe, donde se crea el sistema de educación con políticas, normas, organización para dar atención a los procesos de aprendizaje en idiomas ancestrales y oficiales.

En el Art. 80.- Fines del SEIB se menciona los siguientes fines:

a. El fortalecimiento de la plurinacionalidad y la interculturalidad para lograr el Buen Vivir;

b. El fortalecimiento de la identidad, lengua y cultura de las nacionalida- 
des y pueblos indígenas;

c. El fomento, desarrollo y fortalecimiento de los sistemas de vida de las comunas, comunidades, pueblos y nacionalidades;

d. La recuperación, desarrollo y socialización de la sabiduría, el conocimiento, la ciencia y la tecnología de los pueblos y nacionalidades ancestrales;

e. El impulso de una educación de calidad integral, articulada con la producción, la investigación, la ciencia y los saberes ancestrales;

f. La recuperación, desarrollo y fortalecimiento de los valores propios de las comunas, comunidades, pueblos y nacionalidades;

g. La formación de personas con identidad propia, con un nivel científico que conviva con los avances tecnológicos y los saberes de otros pueblos.

Como se puede notar, la atención a la riqueza cultural del país en ámbitos de educación cuenta con los amparos pertinentes para que se ejecute cualquier acción. Sin embargo, la puesta en práctica de la interculturalidad como un derecho es aún un reto, debido a que las problemáticas particulares de cada región y grupo humano piden enfrentar de manera novedosa y diferente el tema de la comunicación en la diversidad que, si no se toma en cuenta, lo que empieza como ideas propuestas en otros contextos y llenas de buena voluntad puede terminar como hechos que refuerzan esquemas discriminatorios.

A la hora de buscar la forma en que se ejecutan estas disposiciones, se llega a la reflexión sobre cómo confluye el respeto a las diversidades o cómo se interculturaliza la educación. Se conoce que toda la trayectoria de educación indígena a nivel de región es producto de considerar que "los planteamientos étnicos de una educación diferenciada se apoyan en una demanda que aparece en forma constante en el curso de los movimientos indígenas" (González, p. 114). Sin embargo, a la hora de buscar esa diferenciación nos encontramos con múltiples realidades donde se puede sentir y observar encuentros, pero también desencuentros de esa multiculturalidad.

Autores como Velasco (2016), ya vislumbran que la educación formal está influida por fundamentos y orientaciones que la hacen altamente proclive a la producción y reproducción del racismo. El hecho de desarrollar el plan curricular correspondiente favorece la discriminación de los grupos étnicos considerados como "débiles" sin necesidad de identificar contenidos racistas explícitos o implícitos dentro de la educación formal. 


\section{Encuentros y desencuentros}

\section{Identificación de encuentros a la hora de producirse un hecho pedagógico}

Los encuentros observados en la investigación se dan al momento de compartir espacio y tiempo, los que permite la relación y el diálogo de los diferentes actores.

En la escuela Ika se evidencia la presencia de profesores y estudiantes kichwa, shuar, waorani, mestizos, que se encuentran: a la hora de compartir el espacio, de interactuar entre ellos en las actividades cotidianas de la escuela y de ser parte de las reuniones de padres de familia. Se puede también mencionar como encuentro algunos matrimonios, sobre todo entre kichwas y waorani produciéndose un mestizaje.

En la escuela, entre los niños se evidencia la presencia de juegos compartidos que permiten el diálogo de culturas en un ambiente armónico. Lo mismo podríamos decir que sucede en el bus de la petrolera que es utilizado por las comunidades como medio de transporte. Los niños disfrutan del recorrido en el que se intercambia bromas, historias, noticias, y este se convierte en un espacio de armonización e intercambio.

En entrevista realizada a estudiantes de Tránsito Amaguaña, al referirse a la vivencia de la educación intercultural, lo plantean de la siguiente manera: "Como una escuela donde compartimos diferentes identidades, conocemos lo que no conocíamos. Por ejemplo, yo soy de Cotopaxi y conozco a las personas que son de Chimborazo que tienen diferente cultura que la de nosotros".

\section{Identificación de desencuentros a la hora de producirse un hecho pedagógico}

Sin embargo, los desencuentros superan a los encuentros, ya que el reconocimiento "del otro" se torna forzado, como se evidencia en el CECIB Ika. La presencia de docentes que pertenecen a otra cultura desconocen la cultura y la lengua del lugar donde se construye el hecho pedagógico, y se conduce a la imposición de una segunda cultura al forzar a los estudiantes para que adopten palabras, conductas, pensamientos, actitudes que les son ajenas y que les son carentes de significado para su contexto.

Para Krainer (2016), la figura del maestro "en cualquiera de los sistemas educativos se convierte en un transmisor de conocimientos, pero sobre todo de valores y cultura. Por esto es necesario un espacio para compartir entre el mundo indígena y el mestizo, en condiciones de respeto y equidad, sino no habrá interculturalidad" (p. 30).

Las condiciones de respeto y equidad, tan importantes dentro de los 
propósitos citados de la educación intercultural, no se forjan en la escuela. Por citar un ejemplo de las observaciones realizadas dentro de la investigación, se evidencia que dentro de la cultura waorani se vive el momento: el aquí y el ahora cobran importancia en su vida cotidiana. Este hecho, además, les proporciona una libertad que podría dejar de lado la planificación. La entrevista realizada a un waorani de la comunidad lo evidencia cuando afirma: "comemos cuando tenemos hambre y ahí vamos a cazar, dormimos cuando tenemos sueño, no hay hora para comer ni dormir".

De esta forma, el hecho de la asistencia del niño a la escuela, donde se toma muy en cuenta los horarios, se constituye en una imposición de una forma diferente de vida. Los niños y sus familias tuvieron que interiorizar que para asistir a la escuela tienen que levantarse a tales horas, que el bus les recoge en un lugar a una determinada hora, $y$, así, su educación les impone un horario y una cultura a la que no están acostumbrados. Ahora, si planteamos para niños waorani el hecho de vestir un uniforme hay que considerar que ellos habitan en un ambiente que supera los 38 grados centígrados y este hecho significa algo descontextualizado. Se muestra, así que el primer año de nuestra investigación el uniforme consistía en un calentador y un par de zapatos, prenda que en lugar de proteger al niño le causa inconvenientes a la hora de correr, jugar, trepar por los palos o troncos de árboles. El segundo año, se convirtió en algo más fresco pero con unos adornos dorados que nada tienen que ver con su cultura. El Ministerio de Educación reparte uniformes para todas las instituciones y la sola mención de la palabra "uniforme", refleja un mensaje: uniformar a la educación y excluir a la diversidad.

Al evidenciar en las entrevistas realizadas a estudiantes de Tránsito Amaguaña se coteja que algunos docentes son kichwa hablantes y utilizan el kichwa (lengua materna de los estudiantes). Así también, en el contenido de la clase se evidencian temas culturales. Al respecto, un estudiante refiere "los profes nos cuentan cosas, si nos tienen paciencia nos cuentan su vida y las costumbres de antes" y otro afirma: "bailamos música tradicional, como de la mama Tránsito, Cuyaraimi, Jacu jacu".

Al comparar con la realidad del CECIB IKA, en las observaciones realizadas en el aula de clase se puede evidenciar que los profesores no conocen el waotededo (lengua materna de los estudiantes) y tampoco lo utilizan de manera adecuada. Sin embargo, en una de las aulas hay carteles en waotededo y español.

A pesar de esto, en ninguno de los centros se constata resultados que 
evidencien el empoderamiento de su cultura, de tal manera que lleve a los niños a identificarse con ella. Así, en el CECIB IKA, los profesores reportan que los niños pequeños hablan en waotededo pero los mayores ya no lo quieren hablar y tampoco quieren hacer o saber cosas acerca de su cultura. En Tránsito Amaguaña un estudiante, al referirse a la educación intercultural bilingüe afirma: "Es donde enseñan el kichwa también aprendemos a hablar, antes hasta los $7 \mathrm{u}$ 8 años yo si hablaba, pero me he olvidado. Entiendo, pero no hablo. Mis papás si hablan, cuando me dicen yo contesto en español".

Los waorani daban mucha importancia a que sean los ancianos quienes enseñen a sus hijos las actividades diarias. Los ancianos motivaban, mediante el canto, a los pequeños a trabajar. La observación permitía la imitación y la comprensión de los ciclos de la selva, cuando recolectar frutos, dónde y cómo cazar o pescar, el manejo de la chacra, actividades que ahora no se dan debido a que los niños ya no acompañan a los ancianos, sino que van a la escuela. Esto trae consigo una noción de cambio de vida. En este sentido, un desencuentro posible es el proceso de ingreso a la escuela a edades muy tempranas en las que los niños normalmente van adentrándose a las actividades de su cultura que fortalecen valores, actitudes, prácti- cas, etc. Aquí se fortalece o se debilita la cultura de un pueblo.

Por otro lado, los docentes que no están familiarizados con la cultura dominante del país, tienen que "intentar acoplarse" a las exigencias y cumplimientos de procesos para demostrar la efectividad de los sistemas educativos. Es el caso del manejo del currículo, del vaciado de matrices de planificación, reporte de actividades, etc. Esto causa dificultades porque se desconoce el significado de estos requisitos, se ignora el manejo tecnológico o no se cuentan con herramientas informáticas, y. en caso de existir, no les son herramientas familiares a los docentes que trabajan en el área rural.

Finalmente, el desconocimiento por parte de los docentes sobre los principios de la interculturalidad lleva a que se haga todo únicamente con "sentido común o con buenas intenciones". Observamos desencuentros a la hora de manejar horarios, espacios, maneras de saludar, exigencia en cuanto a la vestimenta, disciplina, etc.

Empero, el principal desencuentro se esboza en la dificultad de comunicación entre los miembros de la comunidad educativa: profesores, autoridades, padres de familia y la comunidad. Además, se observa que hay varios autores y protagonistas que tienen cabida en la escuela, la Fundación Repsol, La Estación 
Científica Yasuní y grupos de voluntarios e investigadores, cada uno con su propia idea de lo que se debe o no se debe hacer para mejorar la educación en la escuela IKA.

Apple y Beane, 2005; Bolívar, 2000; Flecha y Tortajada, 1999; Jiménez y Pozuelos, 2001; Alcalde y otros, 2006 citados en Estivalis, M. L., Martí, J. A. T., \& Ciges, A. S. (2016) afirman que "La literatura educativa señala la implicación y participación familiar y comunitaria como uno de los factores que, adecuadamente potenciados y optimizados, contribuyen de forma significativa al éxito escolar y a propiciar una mejora de la igualdad de oportunidades en la escuela" (p. 15).

A este respecto, tanto en el CECIB IKA como en Tránsito Amaguaña, los profesores afirman que son pocos los padres de familia que asisten a la escuela y colaboran con las labores en ella desempeñadas. De esta forma, es aún más difícil que sus aportaciones y críticas a este sistema puedan ser escuchadas, lo que entorpece la diversidad étnica y cultural para alcanzar una mejora comunitaria.

\section{Comparando dos realidades en un solo propósito: la educación}

Lo que merece manifestar en este artículo es que de las dos realidades observadas hay manifestaciones diferentes y similares. Al comparar las experiencias de CECIB IKA y Tránsito Amaguaña, se evidencia una diferencia significativa en los profesores quienes en el primer caso no coinciden con la etnia a la que pertenecen los niños. Se sienten frecuentemente descalificados por otros actores y no reconocidos por la comunidad. En su afán de cumplir con la normativa chocan con los elementos de la cultura, mientras que en el otro caso los profesores y los alumnos pertenecen a la nacionalidad kichwa, por lo tanto, el proceso de encuentro cultural fluye de mejor manera esto se debe al encuentro de cosmovisiones propios de este grupo.

Cabe recalcar que la educación intercultural bilingüe surge en el país por la lucha del pueblo kichwa, quien al momento cuenta con mayor reconocimiento a nivel nacional en cuanto a educación se refiere. Se encuentra mayor cantidad de textos escolares en kichwa y el currículo fue adaptado a su cultura con anterioridad que el currículo presentado para la nacionalidad waorani. Los kichwas cuentan con docentes preparados con títulos de licenciatura, en cambio son muy pocos los docentes waorani.

En la investigación se puede notar que en Tránsito Amaguaña se evidencia la existencia de textos y material didáctico en kichwa. En cambio, la falta de material en waotededo resalta en el CECIB Ika. Por otro lado, la adaptación al currículo realizado en kichwa, a nuestro 
parecer toma más en cuenta su cultura e idioma que la adaptación revisada y adaptada al waotededo en el que se incluye, por ejemplo, la minga como parte fundamental a ser desarrollada dentro de la educación, siendo esta práctica propia de los kichwas y no de los waorani.

\section{CONCLUSIONES}

La investigación realizada nos conduce más hacia reflexiones que a conclusiones, porque las conclusiones son desalentadoras en cuanto al pretendido objetivo de la Educación Intercultural Bilingüe. Concluimos que falta mucho por trabajar para alcanzar un reconocimiento significativo a la diversidad como enriquecedora de un proceso educativo. Es necesario tomar en cuenta otras perspectivas y no solo la mirada de la sociedad dominante o mayoritaria que ha tratado de enmarcar toda la riqueza y diversidad cultural dentro de un solo currículo nacional.

La educación intercultural en cualquier contexto que se lo quiera ejecutar trabaja para cumplir con el objetivo general de "combinar las competencias, habilidades y conocimientos indígenas con las competencias, habilidades y conocimientos escolares convencionales tales que figuran en los currículos nacionales" (Gaschè, 2008, p. 280).

Se encontró, con la investigación, más desencuentros que encuentros. Es desalentador ver cómo, a través de la educación intercultural bilingüe, las instituciones educativas están contribuyendo al desconocimiento y debilitamiento de los logros de los movimientos indígenas y su reconocimiento como integrantes de una sociedad diversa.

Pensamos que se debe trabajar para construir los principios de la interculturalidad e impulsar la formación de profesores que conozcan, valoren y respeten cada uno de los contextos donde se ejerza la educación, y que se propicie la participación de la comunidad y se enriquezcan de ella para que se conviertan en los conductores de los procesos educativos, ya que ellos conocen y viven la filosofía de vida de las nacionalidades y pueblos reconocidos en el país. Con esto estaríamos criticando las decisiones tomadas en el gobierno anterior, el cual tomó la decisión de cerrar a los institutos pedagógicos para docentes interculturales bilingües, por no haber reunido los requisitos necesarios para estar en la dinámica de la educación nacional.

Finalmente, se debe hacer investigaciones más profundas en temas de 
educación intercultural, pues, se presta para reflexionar en aquello que menciona textualmente Gunth Dietz (2012) "al estudiar fenómenos de interculturalidad es indispensable analizar la relación que en estas situaciones se establece entre las diferencias intraculturales existentes al interior de un grupo, y las diferencias interculturales, las que separan o identifican un grupo de otro" (p. 101).

La realidad de cada contexto educativo es diferente, como es diferente cada grupo humano, cada etnia, cada cultura. Por esto, se pretende una educación que promueva la construcción del conocimiento desde la participación activa y el compromiso de la comunidad para descubrir las propias necesidades e intereses que conducirán a la visualización de los cambios requeridos.

Este proceso más que requerir normas estandarizadas, currículos nacionales, pruebas unificadas, que tratan de enmarcar a todos como idénticos, con los mismos intereses, y necesidades, debe valorar las diferencias, analizar las causas y efectos de sus problemas parti- culares y determinar las fortalezas y debilidades para la búsqueda de soluciones propias para cada contexto que permitan a la vez determinar formas de participar de una sociedad incluyente que se enriquezca de estos aportes y proporcione riqueza.

Como afirma Estivalis, et al., () "Las múltiples necesidades detectadas requerían una acción coordinada, comprometida con el cambio global, pero dando pequeños pasos con una estrategia consensuada y esfuerzos compartidos" (p.)

Para finalizar, se considera necesario tener una corresponsabilidad social y educativa, en el trabajo que se efectúa con estos grupos étnicos, entre los órganos de gobierno, como Ministerio de Educación, Ministerio de Inclusión Económica y Social, etc., las instituciones y organizaciones que trabajan geográficamente cerca de estas poblaciones, y los actores del hecho educativo, solo de esta manera se podrá pensar en vivir la interculturalidad donde existan más encuentros que desencuentros. 


\section{BIBLIOGRAFÍA}

Amani, C., \& Gómez Lara, J. (2004). La escuela intercultural: regulación de conflictos en contextos multiculturales: concienciación, negociación, confrontación. Ministerio de Educación y Ciencia, Secretaría General Técnica.

Berteley, María, et al. Educando en la Diversidad. 2008. Quito Ecuador. Abya Yala

CONSTITUCIÒN DE LA REPÜBLICA DEL ECUADOR. http://www.inocar. mil.ec/web/images/lotaip/2015/ literal_a/base_legal/A._Constitucion_republica_ecuador_ 2008constitucion.pdf

CONVENIO 169 OIT. http://www.lo.org/ wcmsp5/groups/public/---americas/---ro-lima/documents/publication/wcms_345065.pdf

Dietz, Gunther. 2012. Multiculturalismo, interculturalidad y diversidad en educación. Una aproximación antropológica. México

Estivalis, M. L., Martí, J. A. T., \& Ciges, A. S. (2016). La escuela en el barrio. Cartografiando las necesidades de cambio socioeducativo. Aularia: Revista Digital de Comunicación, 5(2), 13-20.

Gasché, Jorge. 2008.Niños, maestros, comuneros y escritos antropológi- cos como fuentes de contenidos indígenas escolares y la actividad como punto de partida de los procesos pedagógicos interculturales: un modelo sintáctico de cultura, en Educando en la diversidad. Quito Ecuador. Abya Yala.

Hidalgo Hernández, V. (2017). Cultura, multiculturalidad, interculturalidad y transculturalidad: Evolución de un término. Recuperado de: http://pedagogia.fcep.urv. cat/revistaut/revistes/juny05/article04.pdf

Krainer, A., \& Guerra, M. (2016). Interculturalidad y educación. Desafíos docentes. Quito: FLACSO. Unidad Editorial.

LEY ORGÁNICA DE EDUCACIÓN INTERCULTURAL. Ministerio de Educación del Ecuador. 2011. Quito Ecuador.

UNESCO. Directrices de la Educaciòn Intercultural. http:// unesdoc.unesco.org/images/0014/001478/147878s.pdf

Velasco, Saúl. (2016). Racismo y educación en México. Nueva Época: Revista Mexicana de Ciencias Políticas y sociales, Universidad Autónoma de México, 61(226), 379-408. 
Entrevistas:

Profesor 1: Profesor Tránsito Amaguaña, entrevista realizada por Enrique Contreras, 14 octubre, 2016.

Profesor 2: Profesor Tránsito Amaguaña, entrevista realizada por María Angélica Arroyo y Lorena Álvarez, 14 octubre, 2016.

Profesor 3: Profesor Tránsito Amaguaña, entrevista realizada por María Angélica Arroyo y Lorena Álvarez, 14 octubre, 2016.

Profesor 4: Profesor Tránsito Amaguaña, entrevista realizada por María Angélica Arroyo y Lorena Álvarez, 14 octubre, 2016.

Estudiante 1: varón 17 años, trabaja en la construcción, estudiante en Tránsito Amaguaña, entrevista realizada por Alexandra Yépez y Shadira Procel, 14 octubre 2016.
Estudiante 2: mujer 17 años, vive en unión libre, no trabaja, estudiante en Tránsito Amaguaña, entrevista realizada por Alexandra Yépez y Shadira Procel, 14 octubre 2016.

Estudiante 3: mujer 15 años, estudiante en Tránsito Amaguaña, entrevista realizada por Alexandra Yépez y Shadira Procel, 14 octubre 2016.

Estudiante 4: mujer 12 años, estudiante en Tránsito Amaguaña, entrevista realizada por Alexandra Yépez y Shadira Procel, 14 octubre 2016.

Estudiante 5: mujer 12 años, estudiante en Tránsito Amaguaña, entrevista realizada por Alexandra Yépez y Shadira Procel, 14 octubre 2016.

Estudiante 6: mujer 12 años, estudiante en Tránsito Amaguaña, entrevista realizada por Alexandra Yépez y Shadira Procel, 14 octubre 2016. 